\title{
ELECTRICAL NOISES REDUCTION IN NANOPORES EXPERIMENTS BASED ON CONSENSUS FILTER
}

\author{
Bingyong Yan ${ }^{\mathrm{a}}$, Haixu Cui ${ }^{\mathrm{a}, *, \mathbb{D}}$, Jiale Zhou ${ }^{\mathrm{b}}$ and Huifeng Wang ${ }^{\mathrm{a}}$ \\ ${ }^{a}$ School of Information Science and Engineering, East China University of Science and Technology, Shanghai, 200237, P. R. China \\ ${ }^{\mathrm{b}} \mathrm{S}$ chool of Chemical and Molecular Engineering, East China University of Science and Technology, Shanghai, 200237, P. R. China
}

Recebido em 17/12/2019; aceito em 20/04/2020; publicado na web em 04/06/2020

\begin{abstract}
Nanopore has developed into an indispensable tool for single molecules experiments, where useful information about the characteristics of DNA/RNA can be obtained from the flowing of ionic current elicited by individual molecules as they traverse a single nanometer-scale aperture. However, due to the relatively high background current noise and low measurement bandwidth, it is difficult to distinguish the molecule-specific signals from the intrinsic noises of the nanopore recording systems, which impeded its application in DNA sequencing. In this paper, we present a novel consensus filter-based noise reduction approach, which considerably improves the accuracy of the noise-filtered current traces and the signal-to-noise ratios (SNRs). The new approach is compared to the conventional Bessel filters and validated by analyzing simulated representative translocation data as well as alpha-Hemolysin $(\alpha-\mathrm{HL})$ nanopore experimental data. It is demonstrated that the high frequency noise generated by the voltage noise of the amplifier and Analog-to-Digital converter circuits (ADC) can be significantly reduced and some hidden short-lived translocation events can be recovered with satisfying accuracy beyond the conventional limitations of the Bessel filters.
\end{abstract}

Keywords: nanopores; consensus filter; DNA sequencing; bessel filter.

\section{INTRODUCTION}

In recent years, nanopore techniques has evolved into an extremely powerful and versatile method for studying electrophysiological properties of single molecules, which has been widely used for analyzing various biomolecules analytes, including oligonucleotides, ${ }^{1-5}$ small molecules,${ }^{6,7}$ peptides,${ }^{6,89}$ proteins, ${ }^{10,11}$ and protein-DNA complexes. ${ }^{12,13}$ In these nanopores, Proteinaceous $\alpha$-HL nanopores have been used extensively for detecting and analyzing reaction mechanisms at single molecule level..$^{14,15}$ More recently, the solid-state nanopores have attracted considerable attentions from researchers owing to their advantages on device integration and flexibility in nanopore size and solution conditions. ${ }^{16,17}$ In nanopore experiments, a voltage is applied on the membrane and the ionic current is affected by the single molecules when passing through a nanopore. As is known to all, one of the most fundamental problems for nanopore experiments is to identify the transient change from the flowing of the ionic current elicited by individual molecules as they traverse a single nanometer-scale aperture. Fast translocation event detection with low noise level might eventually allow for identification of different structures along nucleic acids, such as triple strand structures, individual hairpins, and single bound proteins, etc. ${ }^{18}$ However, there are a large variety of low-frequency noise and high-frequency noise when measuring ionic currents, which limit the sensitivity and reliability of nanopore detection. In practice, the ionic currents measurements have been constrained to bandwidths below $100 \mathrm{kHz}$ due to the comparatively high background noise. ${ }^{19}$ The level of ionic current noise has a significant influence on the temporal resolution for single molecule translocation experiment. It often becomes extremely difficult to distinguish specific current signals from the high background noise in a nanopore system, which limits their application as single molecule detectors. Study of the dominant ionic current noise sources is therefore essential for identification of translocation events and evaluating the performance of the experimental approach. Previous studies showed that there

*e-mail: chx310989457@ foxmail.com are different dominant noises in different frequency region. The low frequency noise is dominated by flicker noise arising from fluctuations in the ionic conductance of the nanopores, which is found to obey Hodge's phenomenological relation..$^{20}$ By curing polydimethylsiloxane on the nanopore support chip, the dielectric noise in solid-state nanopores can be reduced dramatically. ${ }^{21}$ In the moderate frequency regime, the dominant noise comes from thermal noise and white noise in the nanopore, measurement circuit electronics, and non-ideal dielectric materials. The high frequency noise is associated with the electrical noise from the voltage amplifier and Analog-to-Digital conversion circuits. ${ }^{19}$ In a nanopore experiment, translocation events converge in the low frequency regime, while electrical noises appear in the high frequency regime. The electrical noise is generally observed to be substantially greater than the signal fidelity, which highly affects the single molecule detection. In this case, electronic filters have to be employed to suppress these high frequency noises. Owing to the outstanding advantages on whole-band frequency hysteresis, the Bessel filters are utilized in time domain analysis. ${ }^{22}$ However, electronic filters distort the ionic current signals by dilating short pulse signals and reducing pulse height, and limit the bandwidth of the sampled ionic current, which will have a great influence on analyzing the characteristic of single molecules. In practice, the intrinsic electrical noises are incorporated in the current traces even though we utilize electronic filters to suppress high frequency noises. To improve the signal-to-noise ratio of the single molecule ionic current, the noises reduction approaches have attracted considerable attention from researchers. Jacob et al. ${ }^{19}$ proposed an integrated nanopore sensing platform to achieve high SNR and low noises. The existing methods including hidden Markov modeling, ${ }^{23-25}$ support vector machine were also developed to detect and extract translocation events in nanopore experiments..$^{26,27}$

As the measurement instruments have approached physical limits with respect to noises suppression and sampling time resolution, the improvement of signal-to-noise ratio will be a challenging task. On reducing high frequency noise, only a few noise studies were investigated so far. The poor SNR and comparatively high background noise in nanopore experiments compelled us to find novel generalized 
ways to reduce high frequency noise and to extract more relevant information from measured current traces.

In the last decades, cooperative control problems have become one of the most attractive control topics and have received considerable attention. Tremendous research effort has been devoted to the control and cooperation methodologies for distributed systems. ${ }^{28}$ Cooperative control systems utilize appropriate protocols and algorithms for exchanging information between multiple dynamic agents that actuate to accomplish consensus or a common objective in the presence of dynamically changing interaction topology and unreliable information exchange. Therefore, consensus problems are one of the most fundamental collaborative information processing problems in cooperative control. In paper $^{29}$ the authors proposed a distributed filter that makes the nodes of a sensor network reach consensus on shared information, referred to as consensus filter. The analysis of the convergence, noise propagation reduction, and ability to track fast signals are provided for consensus filters. The basic idea for consensus filter is to control each node of a network to converge within a ball with specified radius, and thus achieves the goal of improving the SNR.

In this paper, we propose a novel consensus filter based on noise reduction approach for improving the sensitivity of $\alpha$-HL nanopore sensors. Much of the existing research for nanopore experiments focus on single channel currents recording method, while we utilize multiple channel currents measurements to improve the signal-to-noise ratio of single molecules translocation through $\alpha$-HL nanopores and to dig hidden meaningful information behind the ionic current traces. First, we give a brief analysis of dominant noise sources, showing a mathematical description of the measured ionic current. Secondly, we construct the consensus filter using the model of the measured ionic current. Subsequently, we compare the consensus filter to the conventional Bessel filters by analyzing simulated representative translocation data as well as $\alpha$-HL nanopore experimental data. We demonstrated that the employment of the consensus filter can significantly reduce the high frequency noise generated by the voltage noise in the amplifier and Analog-to-Digital converter circuits. Surprisingly, we find that some hidden short-lived translocation events can be recovered with satisfying accuracy beyond the conventional limitations of the Bessel filters, which shed light on its application in DNA sequencing.

\section{EXPERIMENTAL}

\section{Materials}

$\alpha$-HL (lyophilized powder, from Staphylococcus aureus), decane (anhydrous, $\geq 99 \%$ ) and diethyl pyrocarbonate (DEPC, $99 \%$ ) were purchased from Sigma-Aldrich (St. Louis, MO, USA). 1,2-Diphytanoyl-sn-glycero-3-phosphocholine (chloroform, $\geq 99 \%$ ) was purchased from Avanti Polar Lipids Inc. (Alabaster, AL, USA). All of the oligonucleotides used in this study were synthesized by Invitrogen Life Technologies (Shanghai, China). Ultrapure water (reach a resistivity of $18.2 \mathrm{M} \Omega \mathrm{cm}$ at $25^{\circ} \mathrm{C}$ ) was obtained by the Milli-Q System (EMD Millipore, Billerica, MA, U. S. A.). Buffer solution $\left(1 \mathrm{~mol} \mathrm{~L}^{-1} \mathrm{KCl}, 10 \mathrm{mmol} \mathrm{L}{ }^{-1}\right.$ Tris- $\mathrm{HCl}, 1 \mathrm{mmol} \mathrm{L}^{-1}$ EDTA, $\mathrm{pH}$ 8.0) was prepared before used. Unless otherwise noted, all other chemicals were of analytical grade.

\section{Nanopore experiment and data recording}

Detailed protocol for the nanopore experiments has been described in previous studies. ${ }^{30,31}$ The current flowing across the nanopore was amplified by Axopatch 200B (Axon Instruments, Forest
City, CA, USA) under voltage clamp mode. The amplified currents were subsequently filtered by a 4-pole Bessel low-pass filter. Then the filtered signal was feed to all of the 16 channels of DigiData 1440A hardware (Axon Instruments, Forest City, CA, USA) and converted into digital signals. The signals of 16 channels were recorded by PClamp software (Axon Instruments, Forest City, CA, USA). A home-made consensus filter software was used to load the recorded data into Matlab for further analysis.

\section{RESULTS AND DISCUSSION}

\section{The construction of the system}

Figure 1a shows the schematic of the ionic currents measurements system. The measurement system is composed of the following parts: (1) $\alpha$-HL nanopore. The $\alpha$-HL nanopore acts as a biosensor. We can observe multiple distinct translocation events when a short DNA strand passes through the $\alpha$-HL nanopore. (2) patch-clamp amplifier. A bias voltage is applied across the $\alpha$-HL nanopore using two electrodes and connects the electrodes to patch-clamp amplifier to measure changes in ionic current with picoamp sensitivity. (3) ADC. The ADC can sample the ionic current at sampling rate of $100 \mathrm{kHz}$ and transmit the measured currents data to computer via USB connection port. (4) Consensus filter. We utilize 16 sampling channels to measure ionic current simultaneously, and then input the sampled data to the consensus filter. Actually, the consensus filter acts as a data fusion center and can be realized by using matlab code. (5) computer-aided data analysis system. The computer-aided data analysis system utilizes data processing techniques to analyze the ionic current traces, such as histogram, spectral power density, etc.

\section{Analysis of measurement model}

Figure $1 \mathrm{~b}$ shows the analysis of dominant electrical noise during ionic current measurement when single molecule passes through $\alpha$-HL nanopore. From Figure 1b, we can model the ionic current in time domain as follows:

$$
I(t)=I_{0}(t)+I_{e}(t)+v_{f}(t)+v_{t}(t)+v_{e}(t)
$$

where $I(t)$ is the measured current trace. $I_{0}(t)$ is the base current which is proportional to the voltage applied on the pore electrodes. The reason is that $\alpha$-HL nanopore behaves like a resistor component. $I_{e}(t)$ is the translocation events with different piecewise constant values, it happens when single molecule passes through $\alpha$-HL nanopore. $v_{f}(t)$ is the flicker noise with $1 / \mathrm{f}$ characteristics. $v_{t}(t)$ is the thermal noise and dielectric noise. $v_{e}(t)$ is the electrical noise generated by amplifier and $\mathrm{AD}$ converter circuits.

Ideally, we can classify and analyze hundreds of translocation events by using time distribution of ionic current $I_{0}(t)+I_{e}(t)$. The base current $I_{0}(t)$ reflects the applied voltage due to the resistor like characteristic of the $\alpha-\mathrm{HL}$ nanopores. The conductance of the nanopore depends not only on the conductance of the solution, but also on the dimensions of the nanopore. ${ }^{32}$ Changes of the ambient temperature or the concentration of solution, external radiations will be the major reasons for the slowly drifting of the base current $I_{0}(t)$. When single molecule passes through $\alpha$-HL nanopore, the blockage of aperture will result in temporal changes of the ionic current, which corresponds to the item $I_{e}(t)$, standing for the translocation events. Each translocation event is characterized by the dwell time, amplitude..$^{33}$ The accurate identification of translocation events has a broad range of applications, including DNA and protein sequencing, force spectroscopy, etc. However, it is inevitable that the higher 


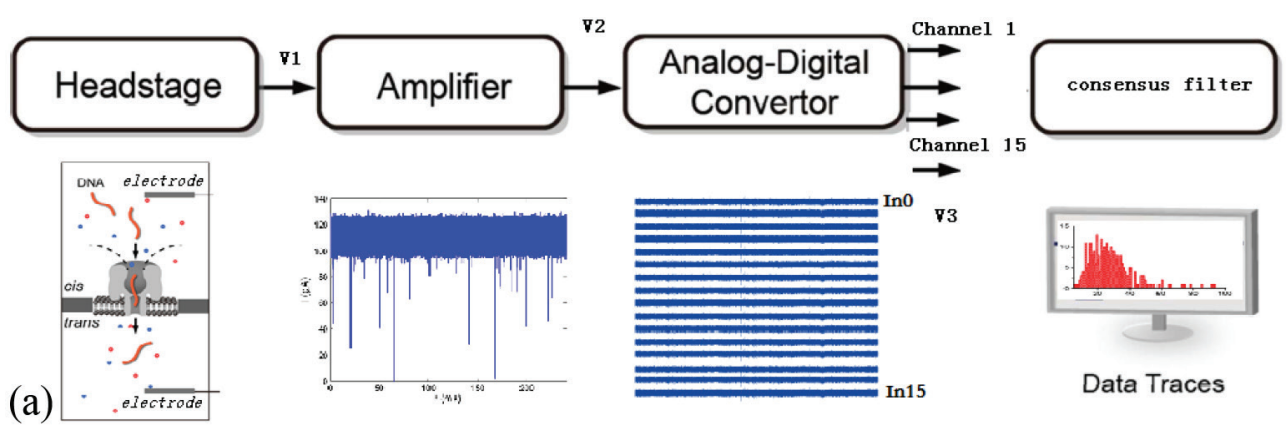

(b)

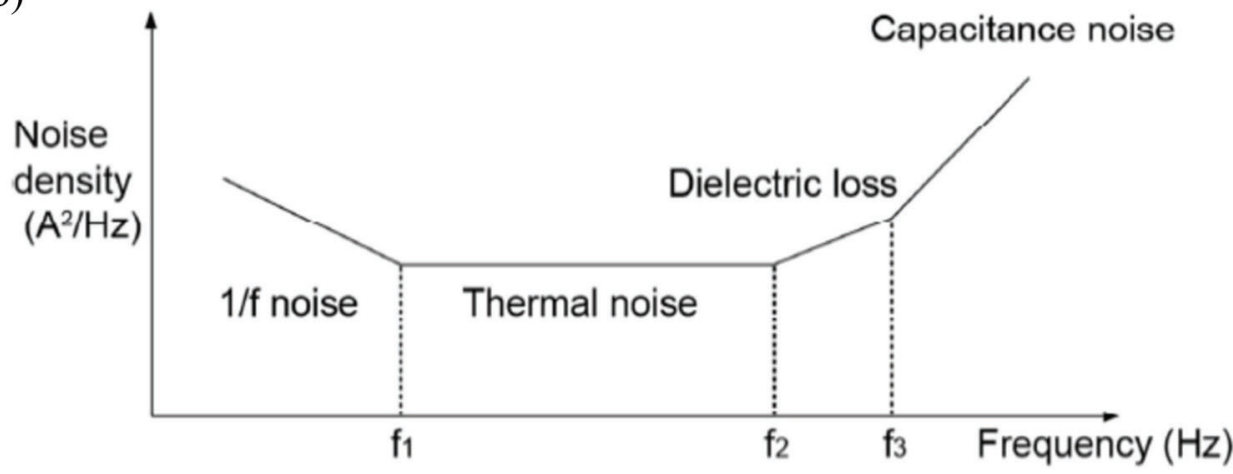

Figure 1. (a) Schematic of the experimental setup (b) The dominant source of noise

background noise is introduced to the measured current by voltage noise in current measurement instruments, which has a great influence on analyzing the characteristics of single molecule, such as $v_{f}(\mathrm{t}), v_{t}(\mathrm{t})$ and $v_{e}(\mathrm{t})$ just mentioned above. Figure 2 a shows measured current trace with translocation events. It is noticeable that the deviation range of the measured base current is from $95 \mathrm{pA}$ to $125 \mathrm{pA}$ approximately, which displays high background noise. Figure $2 \mathrm{~b}$ shows histograms of current traces with translocation events. From Figure $2 b$ we can clearly see that it displays a Gaussian distribution with its center at 113 pA approximately. This is in good agreement with the time domain current deviation. To evaluate the composition of dominant noise in the frequency domain, we take the power spectral density (PSD) of the current traces with translocation events. The PSD has been computed using Welch's averaged modified periodogram. Figure $2 \mathrm{c}$ depicts the noise PSD at $+100 \mathrm{mV}$ for $\alpha$-HL nanopore. It is evident that the high frequency noise converges around $10 \mathrm{kHz}$ while low frequency noise also named flicker noise with $1 / \mathrm{f}$ characteristic is below $10 \mathrm{kHz}$. The high frequency noise above $10 \mathrm{kHz}$ is dominated by electrical noise, which is substantially greater than their organic counterparts. ${ }^{34}$ As a result, the measured ionic current with picoamp sensitivity exhibits a higher background noise. The poor SNR of the measured ionic current will limit their utility as biosensor for single molecule detection.

\section{Construction of consensus filter}

In the previous section, the modeling of the ionic current has been investigated in detail. In this section, we present a novel consensusfilter based method to achieve high SNR and high accuracy by using the ionic current model.

Assume there are $\boldsymbol{n}$ sensors measuring a signal $r(t)$ which is corrupted by zero-mean white Gaussian noises. First, we consider this set of sensors as a connected network with nodes $\boldsymbol{n}$. Then we can construct the mathematical model of the senor network: $p_{i}(t)=r(t)$ $+v_{i}(t),(i=1,2, \ldots, n)$. Where the item $p_{i}(t)$ denotes the measured value of signal $r(\mathrm{t})$ by one sensor. The following dynamic consensus algorithm:

$\dot{x}_{i}(t)=\sum_{j \in N_{i}} a_{i j}\left(x_{j}(t)-x_{i}(t)\right)+\sum_{j \in J_{i}} a_{i j}\left(p_{j}(t)-x_{i}(t)\right),(i, j=1,2, \ldots, n)$

can make $x_{i}(t)$ converge to a ball with radius $\varepsilon$ and noises can be reduced to $\frac{1}{n} \sum_{i=1 \ldots n} v_{i}(t)$. Where $A=\left[a_{i j}\right]$ is a nonnegative adjacency matrix that specify the interconnection topology of the sensors network. $x_{i}(t)$ denotes the current state of node $\boldsymbol{I}$ in the network, and $N_{i}=\left\{i \in V: a_{i j} \neq 0\right\}, J_{i}=N \cup\{i\} . V$ denotes the scale of the network.
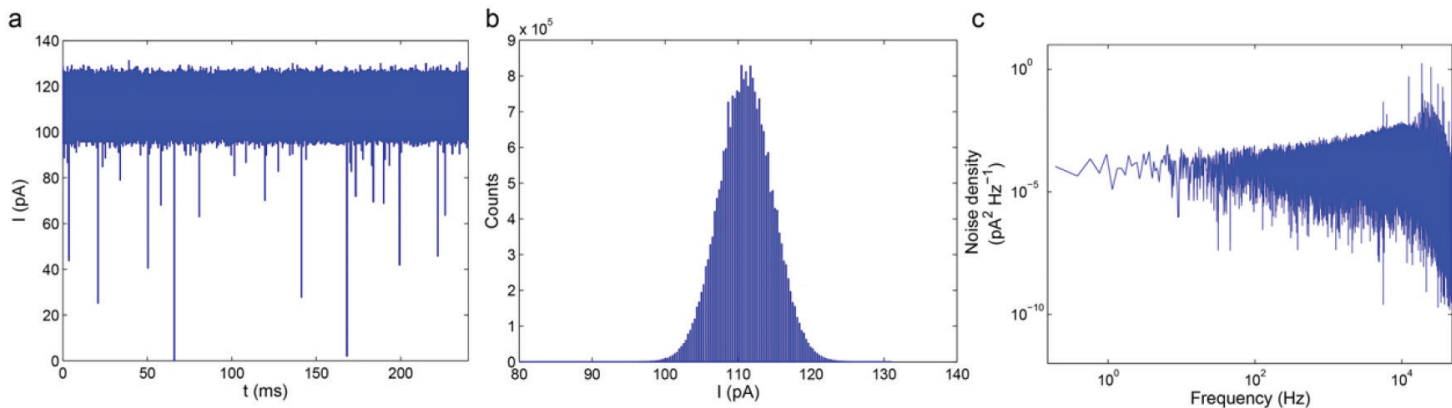

Figure 2. Representative current trace in an $\alpha$-HL nanopore experiment 
Different from the existing filters, the consensus filter can filter high frequency noise, which corresponding to the electrical noise, moreover, it can make the electrical noise reduced by $1 / n$ without distorting the original signal.

The most outstanding difference from the existing current measurement approaches is that we utilize multiple channel ionic current measurements to record ionic current traces. In this experiment, we utilize 16 analog input channels to measure ionic current traces simultaneously. Let $I(t)=\left[I_{i}(t)\right],(i=1,2, \ldots, 15)$ denote the measured current signals. Next, we construct a sensor network of 15 nodes with topology $\mathrm{G}$, and each node is considered as a channel for measuring ionic currents. Assume $\mathrm{G}$ is a graph with matrix $A=$ $\left[\beta_{i j}\right],(i=1,2, \ldots, 16)$ that specifies the interconnection topology of the network. The interconnection topology of the 16 dynamic nodes is shown in Figure 3a. The interconnection of the 15 nodes in Figure $3 \mathrm{a}$ is connected randomly on the initial of the network. Let $\theta=\left[\theta_{i}\right]$, $(i=1,2, \ldots, 16)$ denote the states of each node in graph $G$ with size 16. Then, the consensus filter can be described as follows:

$\dot{\theta}_{i}(t)=\sum_{j \in N_{i}} \beta_{i j}\left(\theta_{j}(t)-\theta_{i}(t)\right)+\sum_{j \in J_{i}} \beta_{i j}\left(I_{j}(t)-\theta_{i}(t)\right)$

where $N_{i}=\left\{i: \beta_{i j} \neq 0\right\}$ is a set of neighbors of node i, $J_{i}=N \cup\{i\}$ is a set of inclusive neighbors of node i. From previously discussion above, we can describe the measured currents as: $I_{i}(t)=I_{0}(t)+I_{e}(t)$ $+v_{f}(t)+v_{e}(t)+v_{t}(t)$ for each analog channel. Our objective is to filter the current signal $I_{i}(t)$ by using consensus filter that takes $I_{i}(t),(i=1$, $2, \ldots, 16)$ as the input and $I_{s}(t)$ as the output with the property that asymptotically all nodes reach an $\varepsilon$ - consensus regarding the value of current $I_{i}(t)$ in all time $t$. The term " $\varepsilon$ - consensus" means that all nodes converge within a ball with radius $\varepsilon$. Figure $3 \mathrm{~b}$ shows that the 16 nodes converge into a ball with radius $\varepsilon$, and the calculation procedures can be found in paper. ${ }^{35}$

To demonstrate the noise reduction ability of consensus filter, we compare the filtered current traces with those by Bessel filter. Figure 4 shows two groups ionic current traces with two representative translocation events, the short-lived translocation event and the long-lasting translocation event respectively. From Figure 4a we can clearly see that there is a representative short-lived translocation event occurring at $\mathrm{t}=2.8 \mathrm{~s}$ (the black curve). To validate the effectiveness of consensus filter, we add the $0.5 \mathrm{dbW}$ Gaussian white noise to the original current trace, as indicated in the grey curve. Obviously, what can be seen in the grey curve is, the short-lived translocation event is hidden as if it is due to noises. Even if we utilize a low-pass four-pole Bessel filter to filter the ionic current trace, the shortlived translocation event is still buried in the background current

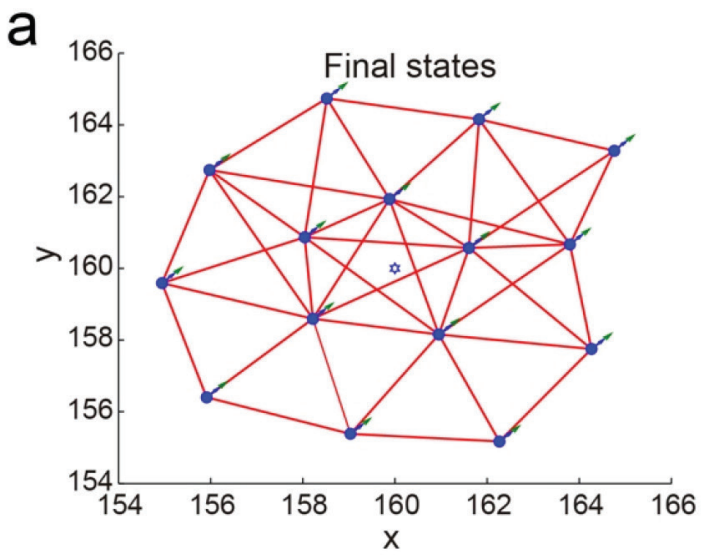

noise, and its behavior is acting like noise (the orange curve). As a consequence, we cannot identify the buried short-lived translocation event effectively. The green curve denotes the filtered ionic current trace by consensus filter. From the green curve, it is noticeable that a short-lived translocation event can be identified at time $\mathrm{t}=2.8 \mathrm{~s}$. Similarly, Figure $4 \mathrm{~b}$ studies the filtered current trace for long-lasting translocation event. These results in Figure $4 \mathrm{~b}$ demonstrate that the consensus filter can filter high frequency noise. As is shown in Figure 4 , the SNR and the accuracy of current traces can be significantly improved for translocation event detection.

\section{Analysis of experimental results}

In Figure 5, we will perform the analysis of consensus filter both in time domain and in frequency domain. At measurement bandwidth set at $100 \mathrm{kHz}$, the noise of the filtered ionic current by consensus filter (the red curve) was substantially lower than that of the unfiltered ionic current (the blue curve) in the condition of no translocation event, owing to the utilization of 16 channels measurements, as indicated in Figure 5a. As seen in Figure 5a, the noise level of ionic current decreases from $6.1 \mathrm{pA}_{\mathrm{RMS}}$ to $1.4 \mathrm{pA}_{\mathrm{RMS}}$, and the $\mathrm{SNR}$ increases from 19 to 27 , before and after the ionic current is filtered by consensus filter. Similarly, Figure 5b investigates the ionic current before and after it is filtered by consensus filter in the presence of translocation events. From Figure 5b, we can clearly see that the noise level of ionic current decreases from $22 \mathrm{pA}_{\mathrm{RMS}}$ to $6 \mathrm{pA}_{\mathrm{RMS}}$, and the $\mathrm{SNR}$ increases from 20 to 27. It is noticeable from Figure 5a and Figure $5 \mathrm{~b}$ that there is a considerable improvement on ionic current accuracy and SNR. Figure 5c and 5d shows the PSD of noise measurement corresponding to the data in Figure $5 \mathrm{a}$ and in Figure $5 \mathrm{~b}$ respectively. Both PSDs have been computed using Welch's averaged modified periodogram. The red curve and blue curve denote the PSD of noise measurement before and after it is filtered by consensus filter in the frequency domain. In the low frequency regime $(<1 \mathrm{kHz})$, the red curve displays hardly any reduction of the noise compared with the blue one. However, in the high frequency regime $(<10 \mathrm{kHz})$, we observe that the noise spectrum of the red curve drops off greatly by comparison with the blue one. We have mentioned above that the noise spectrum in the high frequency regime is dominated by the white noise in the nanopore and amplifier. At frequencies below $100 \mathrm{~Hz}$, it is noticeable that both traces display $1 / \mathrm{f}$ type of noise, which has been investigated previously in nanopores. ${ }^{31}$ The main difference between the red line and the blue line is clearly at the high frequencies around $10 \mathrm{kHz}$. In the high frequencies regime, the dominant noise is the influence of electrical noise, such as interaction of the amplifier's voltage noise. From a comparison of the red line and blue line, it is

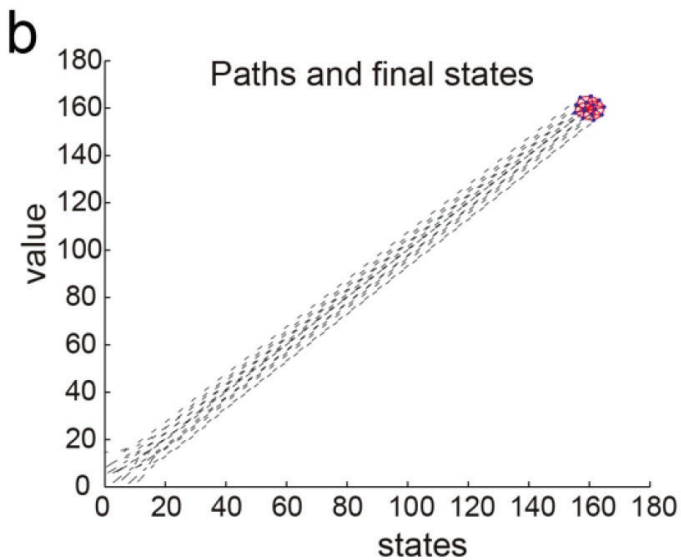

Figure 3. (a) The initial state of each node in a network (b) The node in a network converge into a ball with radius $\varepsilon$ eventually 


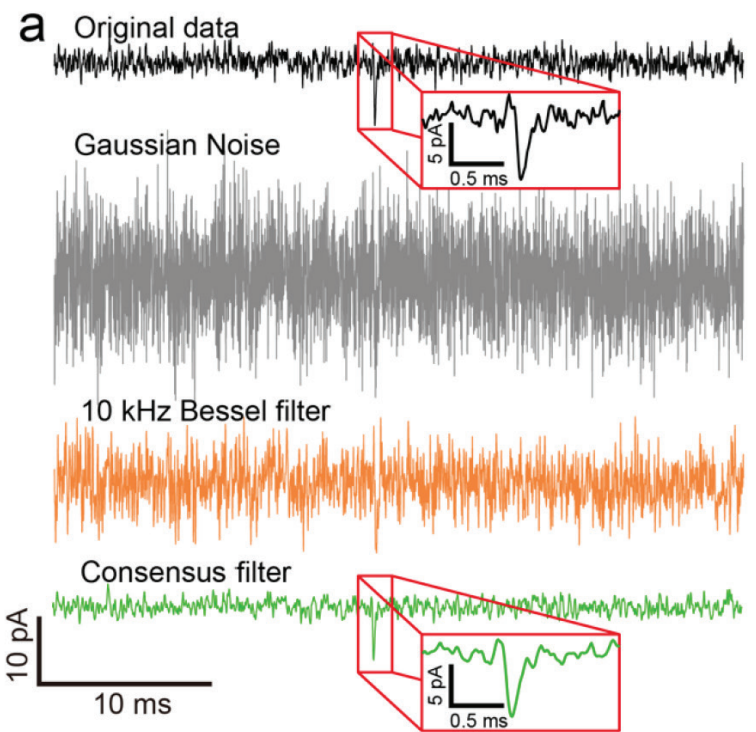

Figure 4. Comparison study between consensus filter and Bessel filter

evident that the high frequency noise has been reduced dramatically by using consensus filter, which is in good agreement with Figure 5a. Therefore, we conclude that the electrical noise is filtered significantly by using consensus filter.

In nanopore experiments, finer temporal resolution allows us to detect short-lived events, which enable us to study comparably small molecules, such as proteins, ${ }^{10,11}$ short RNA or DNA fragments. ${ }^{36}$ As the current noise increases with the enhancement of the ionic current measurement bandwidth, we often utilize electronic filters to suppress high frequencies noise. However, the electronic filter distorts the ionic current signals by dilating short pulse. ${ }^{22}$ One of the outstanding features of the consensus filter is that it can filter the electrical noise without distorting the original signals. We usually utilize the deviation of a data point from the current baseline to identify a pulse. Duo to the presence of measurement noise some short-lived events are hidden as if they are due to noises and therefore can be missed or ignored in computer-aid analysis. During the investigation of consensus filter, a
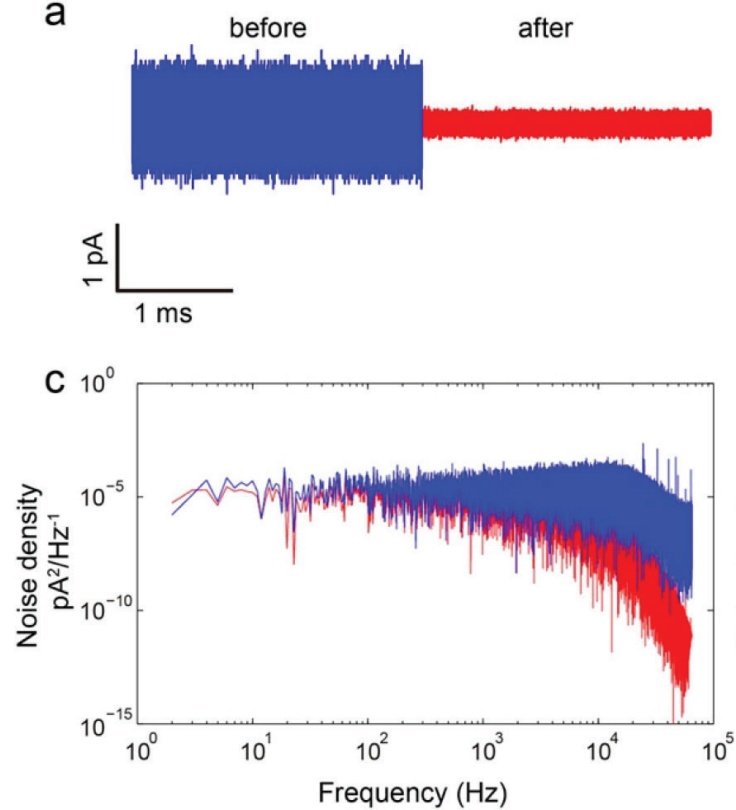

b Original data

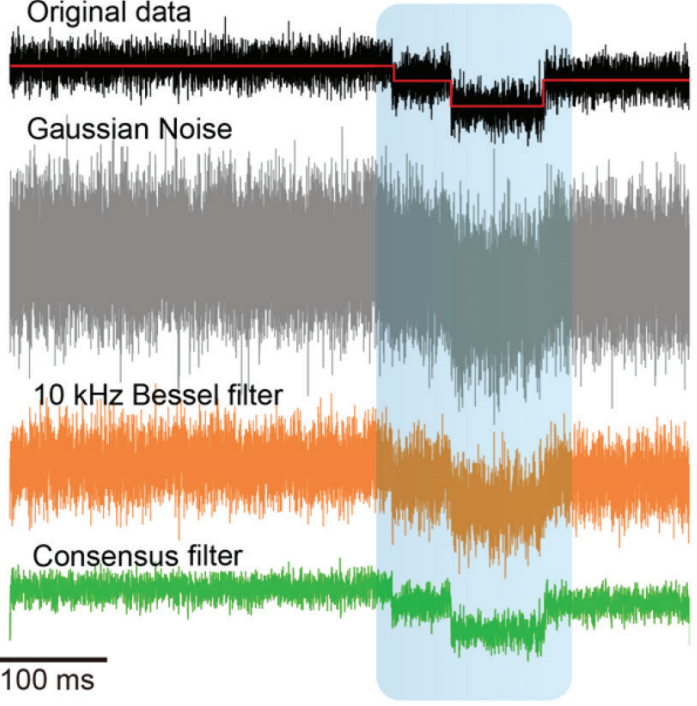

we surprisingly found that the consensus filter has a marked effect on the recovery of the buried translocation events from ionic currents. Figure 6 shows the comparison of filtering results of events with different duration by Bessel filter (the grey curve) and consensus filter (the green curve) respectively. As indicated in the green curve after comparison to the grey curve, aside from the lower noise in time domain, the buried translocation events can be easily identified from the filtered current trace. Figure $6 \mathrm{~d}$ and $6 \mathrm{e}$ shows a scatter plot of translocation events by using Bessel filter and consensus filter respectively. It is noticeable that the dots in Figure 6e moves to the right compared with the dots in Figure 6d. Clearly, events shorter than $10 \mathrm{pA}$ were clearly visible on filtered current traces. In contrast, the Bessel filter has little effect on observing these weak current signals which behave like background noise. Accordingly, translocation events shorter than $10 \mathrm{pA}$ were clearly observed in the filtered current traces. Comparing the two current traces in Figure 6a, 6b, 6c, we can draw the conclusion that the current noise is suppressed within

b
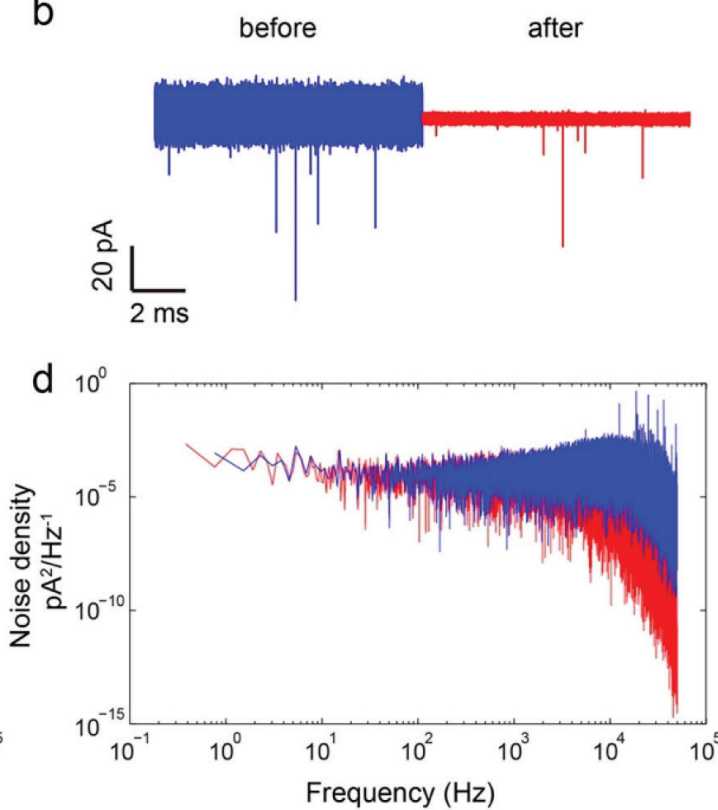

Figure 5. Measurement of current trace before and after the original current trace was filtered with consensus filter 

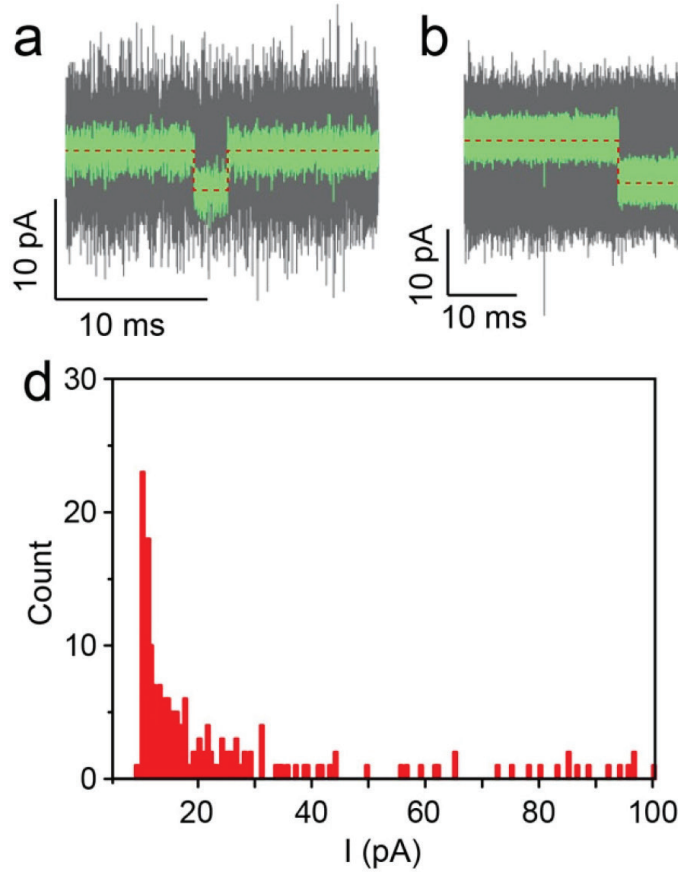

Figure 6. The recovered translocation events by using consensus filter

$5 \mathrm{pA}$ by using the consensus filter based approach, and the range and resolution of singe-molecule characterization with nanopores can be improved dramatically as well. In Figure 7, a detailed design procedure of the consensus filter is given. As indicated in the flow chart, the ionic currents are sampled by using $16 \mathrm{AD}$ converters simultaneously. Each AD can be considered as a node in the consensus filter, then the values of $16 \mathrm{AD}$ converters are sent to formula (1). From formula (1), we can clearly see that the output of the consensus filter can make the electrical noise reduced by $1 / \mathrm{n}$ without distorting the original signal, which is one of the most outstanding feature of the consensus filter compared with Bessel filters.

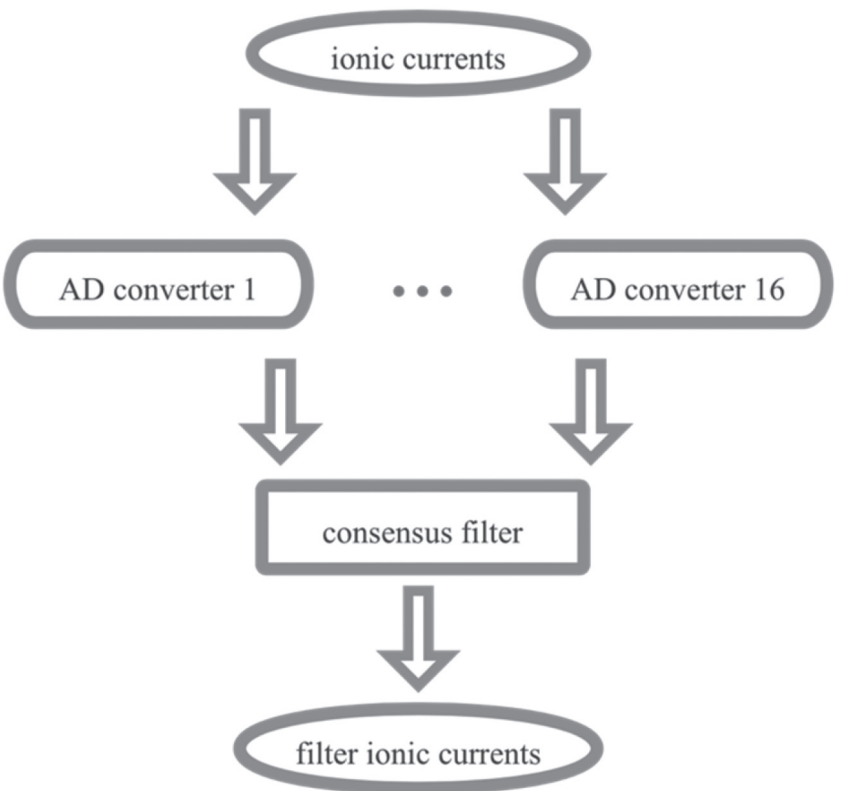

Figure 7. Flow chart of the consensus filter

\section{CONCLUSIONS}

We have investigated the model of the ionic current in nanopore
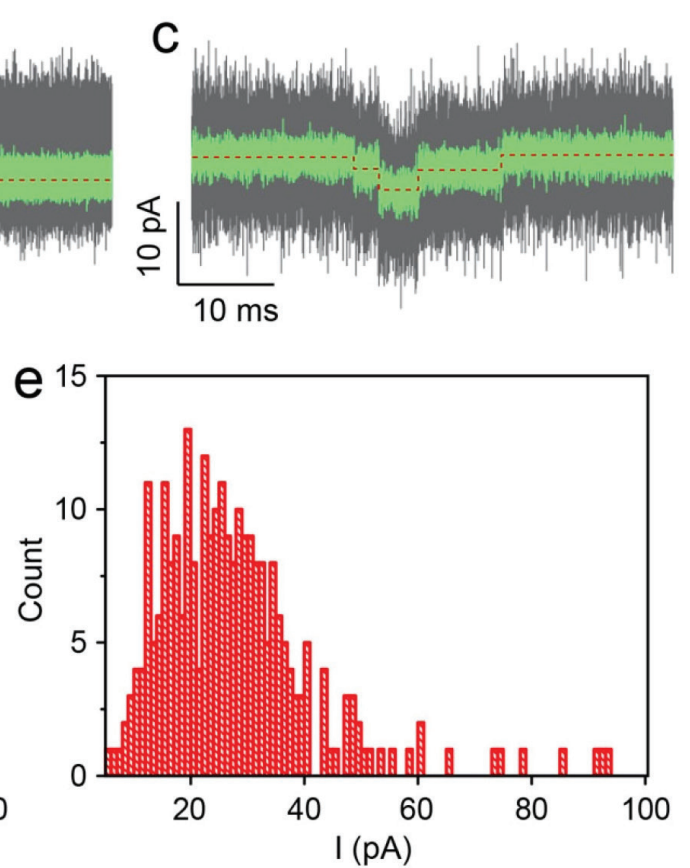

experiments. In this article, we proposed a novel noise reduction approach to improve the signal-to-noise ratio of the ionic current in nanopore experiment. The consensus filter was constructed by using measured ionic currents of 16 analog channels. Different from the existing approach for noise reduction, we utilized the consensus algorithm to control the current value of each channel such that all measured current values converged within a ball with radius $\varepsilon$. Our results demonstrate that consensus filter-based noise reduction approach can improve the signal-to-noise ratio considerably compared with the traditional computing approach. Subsequently, by exploiting different number of analog channels, the research results reveal that the accuracy of signal-to-noise ratio of the ionic current have significant relationship with the sampling channels. The present study provides a general tool to reduce noise in nanopore experiment without changing the hardware and software conditions. Ultimately, we suggest the consensus filter software package can be integrated into the computing software to get the high accuracy of signal-tonoise ratio of the ionic current.

\section{SUPPLEMENTARY MATERIAL}

Supplementary figures are available on http://quimicanova.sbq. org.br in the form of a PDF file, with free access.

\section{ACKNOWLEDGEMENT}

This work was supported by National Natural Science Foundation of China (NO.51407078 and 61773165).

\section{REFERENCES}

1. Ayub, M.; Bayley, H.; Nano. Lett. 2012, 12, 5637.

2. Gu, Z.; Ying, Y. L.; Yan, B. Y.; Wang, H. F.; He, P. G.; Long, Y. T.; Chin. Chem. Lett. 2014, 25, 1029.

3. Carlsen, A. T.; Zahid, O. K.; Ruzicka, J. A.; Taylor, E. W.; Hall, A. R.; Nano. Lett. 2014, 14, 5488.

4. Cao, C.; Ying, Y. L.; Hu, Z. L.; Liao, D. F.; Tian, H.; Long, Y. T.; Nat. Nanotechnol. 2016, 11, 713 . 
5. Chan, C.; Dongfang, L.; Yilun, Y.; Yitao, L.; Acta Chim. Sinica 2016, 74, 734.

6. Wang, H. Y.; Ying, Y. L.; Li, Y.; Kraatz, H. B.; Long, Y. T.; Anal. Chem. 2011, 83, 1746.

7. Kwak, D. K.; Chae, H.; Lee, M. K.; Ha, J. H.; Goyal G.; Kim, M. J.; Chi, S. W.; Angew. Chem., Int. Ed. 2016, 55, 5713.

8. Sutherland, T. C.; Long, Y. T.; Stefureac, R. I.; Bediako-Amoa, I.; Kraatz, H. B.; Lee, J. S.; Nano Lett. 2004, 4, 1273.

9. Mereuta, L.; Roy, M.; Asandei, A.; Lee, J. K.; Park, Y.; Andricioaei, I.; Luchian, T.; Sci. Rep. 2014, 4, 3885.

10. Nivala, J.; Mulroney, L.; Li, G.; Schreiber, J.; Akeson, M.; ACS Nano 2014, 8, 12365.

11. Lepoitevin, M.; Bechelany, M.; Balanzat, E.; Janot, J. M.; Balme, S.; Electrochim. Acta 2016, 211, 611.

12. Bulushev, R. D.; Marion, S.; Radenovic, A.; Nano Lett. 2015, 15, 7118

13. Marshall, M. M.; Ruzicka, J.; Zahid, O. K.; Henrich, V. C.; Taylor, E. W.; Hall, A. R.; Langmuir 2015, 31, 4582

14. Yao, F.; Duan, J.; Wang, Y.; Zhang, Y.; Guo, Y.; Guo, H.; Kang, X.; Anal. Chem. 2014, 87, 338.

15. Bell, N. A.; Keyser, U. F.; Nat. Nanotechnol. 2016, 11, 645.

16. Lee, M. H.; Kumar, A.; Park, K. B.; Cho, S. Y.; Kim, H. M.; Lim, M. C.; Kim, K. B.; Sci. Rep. 2014, 4, 7448.

17. Shekar, S.; Niedzwiecki, D. J.; Chien, C. C.; Ong, P.; Fleischer, D. A.; Lin, J.; Shepard, K. L.; Nano Lett. 2016, 16, 4483.

18. Dekker, C.; Nat. Nanotechnol. 2007, 2, 209.

19. Rosenstein, J. K.; Wanunu, M.; Merchant, C. A.; Drndic, M.; Shepard, K. L.; Nat. Methods 2012, 9, 487.

20. Mohammadi, S.; Pavlidis, D.; IEEE Trans. Electron Devices 2000, 47, 2009.
21. Tabard-Cossa, V.; Trivedi, D.; Wiggin, M.; Jetha, N. N.; Marziali, A.; Nanotechnology 2007, 18, 305505.

22. Pedone, D.; Firnkes, M.; Rant, U.; Anal. Chem. 2009, 81, 9689.

23. Churbanov, A.; Baribault, C.; Winters-Hilt, S.; BMC Bioinf. 2007, 8, S14.

24. Churbanov, A.; Winters-Hilt, S.; BMC Bioinf. 2008, 9, 1.

25. Schreiber, J.; Karplus, K.; Bioinformatics 2015, btv046.

26. Landry, M.; Winters-Hilt, S.; BMC Bioinf. 2007, 8, 1.

27. Eren, A. M.; Amin, I.; Alba, A.; Morales, E.; Stoyanov, A.; Winters-Hilt, S.; Adv. Exp. Med. Biol. 2010, 680, 99.

28. Zhang, H.; Feng, T.; Yang, G. H.; Liang, H.; IEEE Transactions on Cybernetics 2015, 45, 1315 .

29. Olfati-Saber, R.; Shamma, J. S.; Proceedings of the 44th IEEE Conference on Decision and Control, 2005, p. 6698.

30. Gu, Z.; Ying, Y. L.; Cao, C.; He, P.; Long, Y. T.; Anal. Chem. 2015, 87, 907.

31. Yan, B. Y.; Zhen, G. U.; Gao, R.; Cao, C.; Ying, Y. L.; Wei, M. A.; Chin. J. Anal. Chem. 2015, 43, 971

32. Kowalczyk, S. W.; Grosberg, A. Y.; Rabin, Y.; Dekker, C.; Dekker; Nanotechnology 2011, 22, 315101.

33. Clarke, J.; Wu, H. C.; Jayasinghe, L.; Patel, A.; Reid, S.; Bayley, H.; Nat. Nanotechnol. 2009, 4, 265.

34. Tabardcossa, V.; Trivedi, D.; Wiggin, M.; Jetha, N. N.; Marziali, A.; Nanotechnology 2007, 18, 410.

35. Olfati-Saber R.; The joint CDC-ECC '05 Conference 2005, 1, 66398.

36. Smeets, R. M.; Keyser, U. F.; Dekker, N. H.; Dekker, C.; Proc. Natl. Acad. Sci. U. S. A. 2008, 105, 417. 